\title{
Protease-inhibiting, molecular modeling and antimicrobial activities of extracts and constituents from Helichrysum foetidum and Helichrysum mechowianum (compositae)
}

Fanny-Aimée Essombe Malolo ${ }^{1}$, Achille Bissoue Nouga ${ }^{2}$, Antoine Kakam² ${ }^{2}$ Katrin Franke ${ }^{4}$, Lidwine Ngah', Otavio Flausino $\mathrm{rr}^{3}$, Emmanuel Mpondo Mpondo ${ }^{1}$, Fidele Ntie-Kang ${ }^{{ }^{*}}$, Jean Claude Ndom ${ }^{{ }^{*}}$,

Vanderlan da Silva Bolzani ${ }^{3}$ and Ludger Wessjohann ${ }^{4 *}$

\begin{abstract}
Background: Helichrysum species are used extensively for stress-related ailments and as dressings for wounds normally encountered in circumcision rites, bruises, cuts and sores. It has been reported that Helichysum species are used to relief abdominal pain, heart burn, cough, cold, wounds, female sterility, menstrual pain.

Results: From the extracts of Helichrysum foetidum (L.) Moench, six known compounds were isolated and identified. They were 7, 4'-dihydroxy-5-methoxy-flavanone (1), 6'-methoxy-2',4, 4'-trihydroxychalcone (2), 6'-methoxy-2',4dihydroxychalcone -4'-O- $\beta$-D-glucoside (3), apigenin (4), apigenin-7-O- $\beta$-D-glucoside (5), kaur-16-en-18-oic acid (6) while two known compounds 3,5,7-trihydroxy-8-methoxyflavone (12), 4,5-dicaffeoyl quinic acid (13) together with a mixture of phytosterol were isolated from the methanol extract of Helichrysum mechowianum Klatt. All the compounds were characterized by spectroscopic and mass spectrometric methods, and by comparison with literature data. Both extracts and all the isolates were screened for the protease inhibition, antibacterial and antifungal activities. In addition, the phytochemical profiles of both species were investigated by ESI-MS experiments.

Conclusions: These results showed that the protease inhibition assay of $H$. foetidum could be mainly attributed to the constituents of flavonoids glycosides $(3,5)$ while the compound (13) from $\mathrm{H}$. mechowianum contributes to the stomach protecting effects. In addition, among the antibacterial and antifungal activities of all the isolates, compound (6) was found to possess a potent inhibitor effect against the tested microorganisms. The heterogeneity of the genus is also reflected in its phytochemical diversity. The differential bioactivities and determined constituents support the traditional use of the species. Molecular modelling was carried out by computing selected descriptors related to drug absorption, distribution, metabolism, excretion and toxicity (ADMET).
\end{abstract}

Keywords: Compositae, Helichrysum foetidum, Helichysum mechowianum, ESI-MS, Protease inhibition assay, Antimicrobial

\footnotetext{
*Correspondence: ntiekfidele@gmail.com; ndomjefr@yahoo.fr; wessjohann@

ipb-halle.de

${ }^{5}$ Chemical and Bioactivity Information Centre, Department of Chemistry,

University of Buea, P. O. Box 63, Buea, Cameroon

${ }^{1}$ Department of Pharmacy, University of Douala, Douala, P.O. Box 812,

Cameroon

${ }^{4}$ Department of Bioorganic Chemistry, Leibniz Institute of Plant Biochemistry,

Weinberg 3, D-06120 Halle (Saale), Germany

Full list of author information is available at the end of the article
}

\section{Chemistry Central}

(C) 2015 Essombe Malolo et al. This is an Open Access article distributed under the terms of the Creative Commons Attribution License (http://creativecommons.org/licenses/by/4.0), which permits unrestricted use, distribution, and reproduction in any medium, provided the original work is properly credited. The Creative Commons Public Domain Dedication waiver (http://creativecommons.org/publicdomain/zero/1.0/) applies to the data made available in this article, unless otherwise stated. 


\section{Background}

The genus Helichrysum (Compositae) consists of more than 600 species with a major center of distribution in South Africa [1]. Several Helichrysum species have been used in folk medicine of different countries for the protection of post-harvest food [2]. Moreover, Helichrysum species are used extensively for stressrelated ailments and as dressings for wounds normally encountered in circumcision rites, bruises, cuts and sores [3]. It has been also reported that Helichysum species are used to relief abdominal pain, heart burn, cough, cold, wounds, female sterility, menstrual pain [4] and to treat some diseases such as gastric [5-7], gastroduodenal, gastric ulcers and gastritis [8], stomach damage $[9,10]$, acute hepatitis, fever, or oedema [11], diuretic, inflammatory, allergic $[12,13]$. In addition, some of these species have been reported to possess antimicrobially active compounds [14-16].

Chemical studies on Helichrysum species have been carried out by many investigators and the presence of flavonoids, phloroglucinols, $\alpha$-pyrones, coumarins and terpenoid compounds has been reported [17-25]. H. foetidum has been assessed to treat influenza, infected wounds, herpes, eye problems, menstrual pains and to induce trance and possess antifungal properties $[2,26] . H$. mechowianum is used for the treatment of stomach damage, cephalgy $[9,27]$ and possesses ulcerogenic activity [28, 29]. In continuation of these studies, we extended our search for biologically active compounds from Helichrysum species $[17,18]$ to the protease-inhibiting activity of extracts and isolated compounds from Helichrysum foetidum and Helichrysum mechowianum using a fluorescence resonance energy transfer (FRET) protease pepsin inhibition assay as pharmacological model for anti-ulcer compounds [30]. Beside excessive stomach acid and Helicobacter pylori, pepsin is one of the major factors in the pathophysiology of peptic ulcer disease and reflux oesophagitis. In addition, the antibacterial and antifungal effects of both species against Bacillus subtilis and the yeast Cladosporium cucumerinum were evaluated respectively.

The chemical profile of methanol extracts of $H$. mechowianum and $H$. foetidum was investigated. To our knowledge, this is the first report about constituents of $H$. mechowianum. The compounds identified have been reported previously from other Helichrysum species in different compositions.

In order to assess the drug-likeness profiles of the isolated metabolites, low energy computer models were generated and a number of ADMET-related descriptors calculated, with the view of drug metabolism and pharmacokinetics (DMPK) evaluation.

\section{Results and discussion \\ Biological tests}

The methanol leaf extracts of Helichrysum foetidum and Helichrysum mechowianum showed significant activity in the pepsin protease FRET assay while no activity was detected against the serine protease subtilisin (Table 1). The extract of $H$. foetidum exhibited the higher pepsin protease inhibition (37.4 and $35.6 \%$ inhibition at 50 and $25 \mu \mathrm{g} / \mathrm{ml}$ ) (Table 1). Therefore also the previously isolated constituents 1-6 (Fig. 1) from H. Foetidum and 12-13 from $H$. mechowianum were tested. The best results at a concentration of $50 \mu \mathrm{g} / \mathrm{ml}$ were obtained with apigenin-7-O- $\beta$-D-glucoside (5) and $6^{\prime}$-methoxy2 ',4-dihydroxychalcone-4' - $O-\beta$-D-glucoside (3) with a moderate inhibition activity of 46.3 and $37.4 \%$ respectively (Table 1) while 3,5,7-trihydroxy-8-methoxyflavone (12), 4,5-dicaffeoyl quinic acid (13) showed weak activity. These results suggest that the inhibition activity on the aspartate protease observed with $H$. foetidum extract could be mainly attributed to the glycosidic compounds (3) and (5). Contrarily, in the inhibition assay with the

Table 1 Activity (\% inhibition) of Helichrysum crude extracts and major isolated compounds (1-6) in protease inhibition assays using pepsin and subtilisin

\begin{tabular}{|c|c|c|c|c|c|c|}
\hline \multirow{3}{*}{ Sample } & \multicolumn{6}{|c|}{ Inhibition of pepsin (\%) } \\
\hline & \multicolumn{6}{|c|}{ Concentration $(\mu \mathrm{g} / \mathrm{ml})$} \\
\hline & 50 & 25 & 10 & 1 & 0.1 & 0.01 \\
\hline H. mechowianum & 22.8 & 18.8 & 12.0 & 0.3 & 10.9 & 5.4 \\
\hline H. foetidum & 37.4 & 35.6 & 17.8 & nd & 12.6 & 7.1 \\
\hline 1 & nd & 39.5 & nd & -14.2 & -1.5 & 0.4 \\
\hline 2 & nd & 15.9 & 9.2 & -1.4 & nd & 0.3 \\
\hline 3 & 37.4 & 37.4 & 20.1 & 18.2 & 19.4 & 8.0 \\
\hline 4 & 11.6 & 0.2 & nd & 0.2 & 7.8 & 1.8 \\
\hline 5 & 46.3 & 37.2 & 15.1 & 18.6 & -5.3 & -2.9 \\
\hline 6 & 25.0 & -3.6 & nd & -39.0 & -1.2 & -8.0 \\
\hline 12 & 10.5 & 0.7 & $\mathrm{Nd}$ & 0.4 & 6.2 & 2.3 \\
\hline \multirow[t]{2}{*}{13} & 14.7 & 16.4 & 11.8 & 13.7 & 13.1 & 6.1 \\
\hline & \multicolumn{6}{|c|}{ Inhibition of subtilisin (\%) } \\
\hline H. mechowianum & & & -4.8 & 0.2 & 3.0 & -0.1 \\
\hline H. foetidum & & & 0.9 & 2.1 & 4.0 & 3,8 \\
\hline 1 & & & nd & nd & nd & nd \\
\hline 2 & & & -11.6 & -26.2 & 13.2 & nd \\
\hline 3 & & & 9.7 & 4.2 & 6.0 & 3.0 \\
\hline 4 & & & 11.5 & 7.8 & 6.8 & nd \\
\hline 5 & & & 9.2 & 0.3 & 1.0 & -4.8 \\
\hline 6 & & & -11.6 & nd & -5.5 & 16.6 \\
\hline 12 & & & 11.1 & 8.9 & 7.5 & $\mathrm{Nd}$ \\
\hline 13 & & & 8.3 & 5.2 & 7.1 & 3.2 \\
\hline
\end{tabular}


<smiles>COc1cc(O)cc2c1C(=O)CC(c1ccc(O)cc1)O2</smiles>

7,4'-dihydroxy-5-methoxy-flavanone (1)

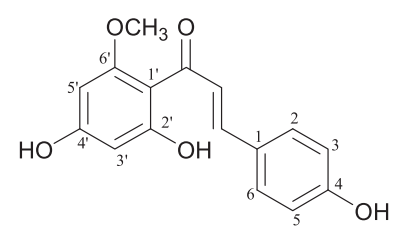

6'-methoxy-2',4,4'-trihydroxy-chalcone (2)

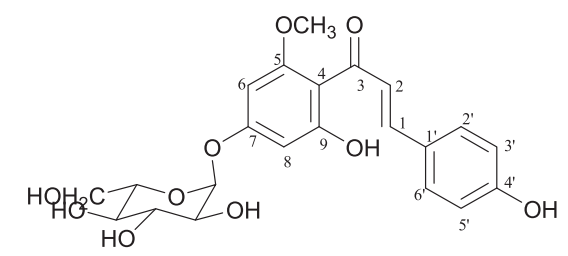

6'-methoxy-2',4-dihydroxychalcone-4'-O- $\beta$ - $D$-glucoside (3)<smiles>O=C1CC(c2ccc(O)cc2)Oc2cc(O)cc(O)c21</smiles>

apigenin (4)

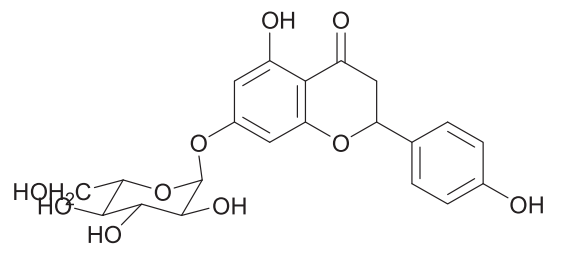

apigenin-7-O- $\beta$ - $D$-glucoside (5)

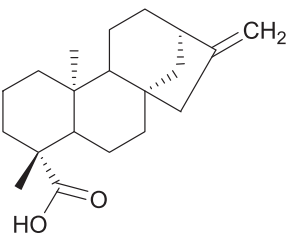

kaur-16-en-18-oic acid (6)<smiles>O=C1C(O)=C(c2ccccc2)Cc2c(O)c(O)cc(O)c21</smiles>

3,5,7 trihydroxy-8-methoxyflavone (12)<smiles>C[C@H](OC(=O)/C=C/c1ccc(O)c(O)c1)OC(C)(C)C</smiles>

Fig. 1 compounds 1-6 isolated from leaves and flowers of H. foetidum and compounds 12-13 from leaves of H. Mechowianum

serine protease subtilisin, neither the crude extracts, nor the isolated substances of both species show significant activity (Table 1). We can conclude that the substances present in the crude extract of $H$. foetidum are selective for aspartate proteases. Observed negative results may be due to the auto-fluorescence debris of subtilisin cleavage of these compounds resulting in fluorescent residues or the absence of bioaffinity interactions between the substances present in the crude extract of $H$. foetidum with the serine protease subtilisin [31]. The observed protease inhibiting activity may have mucosal protective effects and therefore may help to reduce peptic ulceration. From the Black birch fungus (Inonotus obliquus), which is used in folk medicine in Russia for the treatment of gastrointestinal tract disorders, also the flavonoidal fraction was shown to possess antiulcerous activity [31].

In addition, the crude extract of both species and all the isolated compounds were subjected to in vitro antimicrobial assay against the reference strains of bacteria Bacillus subtilis and yeast (Cladosporium cucumerinu).

It has been reported that extracts having MIC values below $8000 \mu \mathrm{g} / \mathrm{ml}$ possess some antimicrobial activity [32]. MIC values below $1000 \mu \mathrm{g} / \mathrm{ml}$ are considered noteworthy $[33,34]$. Thus, the crude extract having activities of $1000 \mu \mathrm{g} / \mathrm{ml}$ or lower against all the pathogens studied demonstrated potential anti-infective properties. Compounds having antimicrobial activities less than $64 \mu \mathrm{g} /$ $\mathrm{ml}$ are accepted as having notable antimicrobial activity [33] and those compounds exhibiting activity at concentrations below $10 \mu \mathrm{g} / \mathrm{ml}$ are considered "clinically significant" [32, 33]. According to this observation, the crude leaf and flower extracts of $H$. foetidum showed a significant and concentration dependent growth inhibition of Bacillus subtilis of of $85.4 \%$ at a concentration of $1 \mathrm{mg} /$ $\mathrm{ml}$ and of $21.8 \%$ at a concentration of $0.1 \mathrm{mg} / \mathrm{ml}$ whereas the crude extract of $H$. mechowianum at concentrations of $1 \mathrm{mg} / \mathrm{ml}$ and $0.1 \mathrm{mg} / \mathrm{ml}$ causes a moderate growth inhibition of 36,2 and $29,8 \%$ respectively (Table 2). Likewise, the crude leave and flower extracts of $H$. Foetidum also exhibit antifungal activity against Cladosporium cucumerinu shown by the development of inhibition zones on the bioautography plate. In contrast, extracts of $H$. mechowianum were slightly active against this fungus.

Furthermore, all of the isolated compounds were subjected to in vitro antimicrobial assay. It was interesting to note that compounds (1-6) from H. Foetidum exhibited notable growth inhibition range of 85.0 to $75.0 \%$ against Bacillus subtilis and a range of 70 to $56 \%$ against the yeast Cladosporium cucumerinu at a concentration of $1 \mathrm{mg} / \mathrm{ml}$ whereas compounds (12-13) from $H$. mechowianum showed a moderate growth inhibition range of 40.2 to $30.8 \%$ at $1 \mathrm{mg} / \mathrm{ml}$ against Bacillus subtilis (Table 2). Of all the isolated, compound (6) exhibited the highest sensitivity growth inhibition of Bacillus subtilis of $85.0 \%$ at a 
Table 2 Antimicrobial activity ((\%)of Helichrysum crude extracts and isolated compounds 1-6, 12-13 from H. Foetidum

\begin{tabular}{|c|c|c|c|c|}
\hline \multirow{4}{*}{ Sample } & \multicolumn{4}{|c|}{ Minimum inhibitory concentration ((\%) } \\
\hline & \multicolumn{4}{|c|}{ Concentration $(\mathrm{mg} / \mathrm{ml})$} \\
\hline & \multicolumn{4}{|c|}{ Cladosporium cucumerinum Bacillus subtilis } \\
\hline & \multicolumn{4}{|c|}{10.110 .1} \\
\hline H. mechowianum & 13.8 & 10.4 & 36.2 & 29.2 \\
\hline H. foetidum & 71.3 & 17.7 & 85.4 & 21.8 \\
\hline 1 & 68.5 & & 76.3 & \\
\hline 2 & 70.0 & & 77.8 & \\
\hline 3 & 66.7 & & 78.2 & \\
\hline 4 & 59.6 & & 75.1 & \\
\hline 5 & 56.1 & & 81.6 & \\
\hline 6 & 69.1 & & 85.0 & \\
\hline 12 & NT & & 40.2 & \\
\hline 13 & NT & & 30.8 & \\
\hline AMP & 74.5 & & & \\
\hline $\mathrm{CIP}$ & & & 94.2 & \\
\hline
\end{tabular}

CIP Ciprofloxacin antibacterial standard, AMP Amphotericin-B antifungal standard, nd not detected

concentration of $1 \mathrm{mg} / \mathrm{ml}$ and was found to be the most active component of the crude flower extract of $H$. Foetidum (Table 2).

The results of the work indicate that diterpenoid possess antimicrobial against the gram positive bacterium. This antibacterial activity of $H$. foetidum extract might be associated to the high content of kaurenoic acid (6). This justifies the use of these plants species in folk medicine and corroborated with the previous reports on the antibacterial activities for Helichrysum species [2, 35, 36]. Kaur-16-en-19-oic acid isolated from extract of the Asteraceae (Senecio erechtitoides and Wedelia calendulaceae) was previously shown to possess high inhibitory activity against several bacterial strains $[37,38]$.

\section{Chemical constituents}

The main constituents of both species were characterized by detailed ESI-MS investigations. The combination of LC-MS, MS/MS and FTICR-HRMS allowed the detection of various components simultaneously. The MS experiments show, that $H$. foetidum and $H$. mechowianum possess different chemical compositions. The leaf extract of $H$. foetidum is dominated by the chalcones 2 and $\mathbf{3}$, the flavonoids $\mathbf{4}$ and $\mathbf{5}$ and by diterpenoids [18] whereas main constituents of $H$. mechowianum are quinic acid derivatives with a less prominent bioactivity profile.

A more detailed ESI-MS investigation of the crude extract of Helichrysum mechowianum indicates (Additional file 1) the presence of quinic acid (7, ESI-FTICR-MS: $[\mathrm{M}-\mathrm{H}]^{-}, m / z$ 191.05578, calc. for $\mathrm{C}_{7} \mathrm{H}_{11} \mathrm{O}_{6}^{-}$191.05556, ferulic acid (8, ESI-FTICR-MS: [M - H] $]^{-}, m / z$ 193.05028, calc. for $\mathrm{C}_{10} \mathrm{H}_{9} \mathrm{O}_{4}^{-}$193.05008, chlorogenic acid (9, ESIFTICR-MS: $[\mathrm{M}-\mathrm{H}]^{-}, m / z 353.08751$, calc. for $\mathrm{C}_{16} \mathrm{H}_{17} \mathrm{O}_{9}^{-}$ 353.08726, three isomers of dicaffeoyl quinic acid (10, ESI-FTICR-MS: $[\mathrm{M}-\mathrm{H}]^{-}, \quad m / z$ 515.11949, calc. for $\mathrm{C}_{25} \mathrm{H}_{23} \mathrm{O}_{12}^{-} 515.11895$, and three isomers of methyl derivatives of $10\left(11\right.$, ESI-FTICR-MS: $[\mathrm{M}-\mathrm{H}]^{-}, \quad \mathrm{m} / z$ 529.13539 calc. for $\mathrm{C}_{26} \mathrm{H}_{25} \mathrm{O}_{12}^{-} 529.46950$.

Compounds 7-11 were detected before in other Helichrysum species. Mono- and dicaffeoyl quinic acids are the main constituents of the Mediterranean herb H. italicum $[38,39]$ and are also present in the French $H$. stoechas var. olonnense [40]. Both are used as digestive. A similar compound composition is known for the Artichoke; Cynara scolymus L., which is used for its choleretic, lipid-lowering, hepatostimulating, and appetitestimulating actions [41]. Extracts and constituents of artichoke were also shown to possess antibacterial and antifungal activities, however, Extracts and constituents of $H$. mechowianum showed least efficiency antifungal properties against the yeast Cladosporium cucumerinum. The observed quinic acid derivatives might be responsible for the stomach protecting effects of $H$. mechowianum.

Chromatographic separation of the partitioned extracts of $H$. mechowianum resulted in the isolation of a phytosterol mixture from the $n$-heptane fraction, 3,5,7-trihydroxy-8-methoxyflavone (12, ESI-FTICR-MS: $[\mathrm{M}+\mathrm{Na}]^{+}$ $m / z 323.05297$ calc. for $\left.\mathrm{C}_{16} \mathrm{H}_{12} \mathrm{O}_{6} \mathrm{Na} 323.05261\right)$ from the ethyl acetate fraction and 4,5-dicaffeoyl quinic acid (13, ESI-FTICR-MS: $\quad[\mathrm{M}-\mathrm{H}]^{-}, \quad \mathrm{m} / \mathrm{z} \quad 515.12168$ calc. for $\mathrm{C}_{25} \mathrm{H}_{23} \mathrm{O}_{12}^{-}$515.11950) from the water fraction. The relative composition of the phytosterol fraction was determined by GCMS as campesterol (2\%), stigmasterol (9.3\%), campest7-en-3-ol (61.4\%), chondrillasterol (18.9\%), $\beta$-sitosterol $(61.4 \%)$ and an unidentified sterol (1.2\%). The compounds (12) [42] and (13) [43] were identified by comparison of spectral data with literature data. In addition, the position of the caffeoyl residues in compound (13) was determined by $2 \mathrm{D}$ NMR measurements. In particular, HMBC correlations from $\mathrm{H}-4$ and $\mathrm{H}-5$ of the quinic acid to $\mathrm{C}-9^{\prime}, \mathrm{C}-8^{\prime}$ and $\mathrm{C}^{-7}$ ' of the caffeoyl residues indicates the substitution at position 4 and 5 (Table 3). Since this compound is reported to possess cytotoxic and apoptose inducing activity $[44,45]$, the anticancer activity against the prostate cancer cell line PC-3 was tested. However in concentrations of 50 $\mathrm{nM}$ or $50 \mu \mathrm{M}$ no effect was observed with this cell line (Table 4).

\section{In silico pharmacokinetics assessment}

Many bioactive compounds do not make it to clinical trials because of adverse pharmacokinetic properties. It therefore becomes imperative to access the pharmacokinetic profiles of potential drugs early enough in order to access their potential for further development. A 
Table $3{ }^{1} \mathrm{H}$ and ${ }^{13} \mathrm{C}$ NMR assignments for compounds $(1-5,12)$

\begin{tabular}{|c|c|c|c|c|c|c|c|c|c|c|c|c|}
\hline$\overline{N^{0}}$ & 1 & & 2 & & 3 & & 4 & & 5 & & 12 & \\
\hline & $\begin{array}{l}{ }^{1} \mathrm{H} \text { (DMSO- } \\
\left.\mathrm{d}_{6}\right)\end{array}$ & $\begin{array}{l}{ }^{13} \mathrm{C} \\
(\mathrm{DMSO}- \\
\left.d_{6}\right)\end{array}$ & $\begin{array}{l}{ }^{1} \mathrm{H}(\mathrm{MeOD}+ \\
\left.\mathrm{CDCl}_{3}\right)\end{array}$ & $\begin{array}{l}{ }^{13} \mathrm{C}(\mathrm{MeOD} \\
\left.+\mathrm{CDCl}_{3}\right)\end{array}$ & $\begin{array}{l}{ }^{1} \mathrm{H} \\
\text { (MeOD) }\end{array}$ & ${ }^{13} \mathrm{C}$ & $\begin{array}{l}{ }^{1} \mathrm{H} \\
\text { (DMSO- } \\
\mathrm{d}_{6} \text { ) }\end{array}$ & $\begin{array}{l}{ }^{13} \mathrm{C} \\
\text { (DMSO- } \\
\left.d_{6}\right)\end{array}$ & $\begin{array}{l}{ }^{1} \mathrm{H} \\
\left(\mathrm{CD}_{3} \mathrm{OD}\right)\end{array}$ & $\begin{array}{l}{ }^{13} \mathrm{C} \\
\left(\mathrm{CD}_{3} \mathrm{OD}\right)\end{array}$ & ${ }^{1} \mathrm{H}\left(\mathrm{CDCl}_{3}\right)$ & $\begin{array}{l}{ }^{13} \mathrm{C} \\
\left(\mathrm{CDCl}_{3}\right)\end{array}$ \\
\hline 1 & & & & 128.0 & & 128.0 & & & & & & \\
\hline 2 & $\begin{array}{l}5.32(1 \mathrm{H}, \mathrm{dd}, \\
12.6,2.9)\end{array}$ & 79.0 & $\begin{array}{l}7.49(1 \mathrm{H}, \mathrm{d} \\
8.6)\end{array}$ & 131.0 & $\begin{array}{l}7.51(1 \mathrm{H} \\
\text { d, 8.7) }\end{array}$ & 131.0 & & 164.4 & & 163.0 & & 136.5 \\
\hline \multirow[t]{2}{*}{3} & $2.51(\mathrm{H} 3 \mathrm{a})$ & \multirow[t]{2}{*}{42.3} & \multirow{3}{*}{$\begin{array}{l}6.84(1 \mathrm{H}, \mathrm{d}, \\
8.6)\end{array}$} & \multirow[t]{2}{*}{116.6} & \multirow{3}{*}{$\begin{array}{l}6.83(1 \mathrm{H}, \\
\mathrm{d}, 8.7)\end{array}$} & \multirow[t]{2}{*}{116.6} & \multirow{3}{*}{$\begin{array}{l}6.78(1 \mathrm{H}, \\
\mathrm{s})\end{array}$} & \multirow[t]{2}{*}{106.5} & \multirow[t]{4}{*}{$6.58(1 \mathrm{H}, \mathrm{s})$} & \multirow[t]{2}{*}{103.1} & \multirow{3}{*}{$\begin{array}{l}6.70(1 \mathrm{H}, \\
\mathrm{s}, \mathrm{OH})\end{array}$} & \multirow[t]{2}{*}{144.9} \\
\hline & $2.98(\mathrm{H} 3 \mathrm{~b})$ & & & & & & & & & & & \\
\hline 4 & & 196.3 & & 160.5 & & 160.5 & & 180.4 & & 182.0 & & 175.6 \\
\hline 5 & 3.72 (OMe) & 163.0 & $\begin{array}{l}6.84(1 \mathrm{H}, \mathrm{d} \\
8.6)\end{array}$ & 116.6 & $\begin{array}{l}6.83(1 \mathrm{H}, \\
\mathrm{d}, 8.7)\end{array}$ & 116.6 & $\begin{array}{l}12.96(1 \mathrm{H}, \\
\mathrm{s}, \mathrm{OH})\end{array}$ & 164.94 & & 164.3 & $\begin{array}{l}11.49(1 \mathrm{H}, \\
\mathrm{s}, \mathrm{OH})\end{array}$ & 156.6 \\
\hline 6 & $\begin{array}{l}6.04(1 \mathrm{H}, \mathrm{d}, \\
2.2)\end{array}$ & 104.6 & $\begin{array}{l}7.49(1 \mathrm{H}, \mathrm{d} \\
8.6)\end{array}$ & 131.0 & $\begin{array}{l}7.51(1 \mathrm{H}, \\
\mathrm{d}, 8.7)\end{array}$ & 131.0 & $\begin{array}{l}6.19(1 \mathrm{H}, \\
\mathrm{d}, 2.0)\end{array}$ & 104.8 & $\begin{array}{l}6.83(1 \mathrm{H}, \mathrm{d}, \\
2.1 \mathrm{~Hz})\end{array}$ & 99.9 & $6.33(1 \mathrm{H}, \mathrm{s})$ & 130.4 \\
\hline 7 & $9.60(\mathrm{OH})$ & 167.1 & & & & & $\mathrm{OH}$ & 160.1 & & 157.0 & $\begin{array}{l}6.46(1 \mathrm{H}, \\
\mathrm{s}, \mathrm{OH})\end{array}$ & 155.4 \\
\hline 8 & $\begin{array}{l}5.94(1 \mathrm{H}, \mathrm{d} \\
2.2)\end{array}$ & 95.4 & & & & & $\begin{array}{l}6.48(1 \mathrm{H}, \\
\mathrm{d}, 2.0)\end{array}$ & 99.3 & $\begin{array}{l}6.71(1 \mathrm{H}, \mathrm{d}, \\
2.1)\end{array}$ & 99.6 & $\begin{array}{l}4.05(3 \mathrm{H}, \\
\text { s, OMe) }\end{array}$ & 148.0 \\
\hline 9 & & 162.8 & & & & & & 160.7 & & 161.1 & & 155.4 \\
\hline 10 & & 101.6 & & & & & & 109.3 & & 105.4 & & 130.7 \\
\hline $1^{\prime}$ & & 129.6 & & 106.3 & & 106.3 & & 123.1 & & 121.0 & & 126.9 \\
\hline $2^{\prime}$ & $\begin{array}{l}7.28(1 \mathrm{H}, \mathrm{d} \\
8.6)\end{array}$ & 127.6 & & 165.9 & $\begin{array}{l}7.51(1 \mathrm{H}, \\
\mathrm{d}, 8.7)\end{array}$ & 165.9 & $\begin{array}{l}7.93(1 \mathrm{H} \\
\mathrm{d}, 8.8)\end{array}$ & 129.3 & $\begin{array}{l}7.83(1 \mathrm{H}, \mathrm{d} \text {, } \\
8.8)\end{array}$ & 128.7 & $\begin{array}{l}8.24(2 \mathrm{H}, \\
\mathrm{d}, 7.1)\end{array}$ & 128.8 \\
\hline $3^{\prime}$ & $\begin{array}{l}6.77(1 \mathrm{H}, \mathrm{d}, \\
8.6)\end{array}$ & 115.0 & $\begin{array}{l}5.99(1 \mathrm{H}, \mathrm{d}, \\
2.2)\end{array}$ & 92.2 & $\begin{array}{l}6.31(1 \mathrm{H} \\
\mathrm{d}, 2.2)\end{array}$ & 92.2 & $\begin{array}{l}6.93(1 \mathrm{H}, \\
\mathrm{d}, 8.8)\end{array}$ & 117.0 & $\begin{array}{l}6.92(1 \mathrm{H}, \mathrm{d}, \\
8.8)\end{array}$ & 116.0 & $\begin{array}{l}7.60 \\
(1 \mathrm{H}, \mathrm{m})\end{array}$ & 127.5 \\
\hline $4^{\prime}$ & $\begin{array}{l}9.60(1 \mathrm{H}, \mathrm{s}, \\
\mathrm{OH})\end{array}$ & 157.6 & & 168.2 & & 168.2 & $\mathrm{OH}$ & 162.6 & & 161.5 & $\begin{array}{l}7.49 \\
(1 \mathrm{H}, \mathrm{m})\end{array}$ & 98.2 \\
\hline $5^{\prime}$ & $\begin{array}{l}6.77(1 \mathrm{H}, \mathrm{d}, \\
8.6)\end{array}$ & 115.0 & $\begin{array}{l}5.96(1 \mathrm{H}, \mathrm{d}, \\
2.2)\end{array}$ & 96.9 & $\begin{array}{l}6.24(1 \mathrm{H} \\
\mathrm{d}, 2.2)\end{array}$ & 96.9 & $\begin{array}{l}6.93(1 \mathrm{H} \\
\mathrm{d}, 8.8)\end{array}$ & 117.0 & $\begin{array}{l}6.92(1 \mathrm{H}, \mathrm{d}, \\
8.8)\end{array}$ & 116.0 & $\begin{array}{l}7.60 \\
(1 \mathrm{H}, \mathrm{m})\end{array}$ & 127.5 \\
\hline $6^{\prime}$ & $\begin{array}{l}7.28(1 \mathrm{H}, \mathrm{d}, \\
8.6)\end{array}$ & 127.6 & & 164.2 & $\begin{array}{l}7.51(1 \mathrm{H} \\
\mathrm{d}, 8.7)\end{array}$ & 164.2 & $\begin{array}{l}7.93(1 \mathrm{H} \\
\mathrm{d}, 8.8)\end{array}$ & 129.3 & $\begin{array}{l}7.83(1 \mathrm{H}, \mathrm{d}, \\
8.8)\end{array}$ & 128.7 & $\begin{array}{l}8.24(2 \mathrm{H} \\
\mathrm{d}, 7.1)\end{array}$ & 128.8 \\
\hline $\begin{array}{l}\text { OMe- } \\
5\end{array}$ & 3.72 & 55.4 & & & & & & & & & & \\
\hline $\begin{array}{l}\text { OMe- } \\
6^{\prime}\end{array}$ & & & 3.93 & 56.2 & 3.95 & 56.2 & & & & & & \\
\hline \multirow[t]{4}{*}{$\begin{array}{l}\text { OMe- } \\
8\end{array}$} & & & & & & & & & & & & 61.9 \\
\hline & & & $\begin{array}{l}7.73(1 \mathrm{H}, \mathrm{d} \\
16.0, \mathrm{Ha})\end{array}$ & $125.2(\mathrm{CH}-\mathrm{a})$ & $\begin{array}{l}7.72(1 \mathrm{H}, \\
\mathrm{s}, \mathrm{Ha})\end{array}$ & 125.2 & & & & & & \\
\hline & & & $\begin{array}{l}7.68(1 \mathrm{H}, \mathrm{d}, \\
16.0, \mathrm{H \beta})\end{array}$ & $143.5(\mathrm{CH}-\beta)$ & $\begin{array}{l}7.71(1 \mathrm{H}, \\
\mathrm{s}, \mathrm{H} \beta),\end{array}$ & 143.5 & & & & & & \\
\hline & & & & $\begin{array}{l}193.6(C= \\
\text { O) }\end{array}$ & & $\begin{array}{l}193.6 \\
(C=O)\end{array}$ & & & & & & \\
\hline 1" & & & & & $\begin{array}{l}4.99(1 \mathrm{H} \\
\text { d, } 7.5)\end{array}$ & 103.7 & & & $\begin{array}{l}4.90(1 \mathrm{H}, \mathrm{d}) \\
7.6\end{array}$ & 105.1 & & \\
\hline $2^{\prime \prime}$ & & & & & 4.24 & 71.0 & & & $\begin{array}{l}3.50-3.25 \\
(4 \mathrm{H}, \mathrm{m})\end{array}$ & 74.8 & & \\
\hline $3^{\prime \prime \prime}$ & & & & & 3.61 & 77.2 & & & $\begin{array}{l}3.50-3.25 \\
(4 \mathrm{H}, \mathrm{m})\end{array}$ & 77.5 & & \\
\hline $4^{\prime \prime}$ & & & & & 3.60 & 78.9 & & & $\begin{array}{l}3.50-3.25 \\
(4 \mathrm{H}, \mathrm{m})\end{array}$ & 71.8 & & \\
\hline
\end{tabular}


Table 4 LC/MS data, deprotonated and protonated molecules $(\mathrm{m} / \mathrm{z})$ for peaks, including the retention times (Rt), MS/MS experiments of the constituents found in $\mathrm{MeOH}$ extract of Helichrysum mechowianum and Helichrysum foetidum

\begin{tabular}{|c|c|c|c|c|c|c|c|}
\hline \multicolumn{4}{|c|}{ Helichrysum mechiowianum } & \multicolumn{4}{|c|}{ Helichrysum foetidum } \\
\hline $\begin{array}{l}\text { Rt } \\
(\min .)\end{array}$ & $\begin{array}{l}\text { HR-MS }(m / z) \text { from } \\
{[\mathrm{M}-\mathrm{H}]^{-}}\end{array}$ & $\begin{array}{l}\text { Molecular } \\
\text { formula }\end{array}$ & $\begin{array}{l}\text { Identified compounds by } \\
\text { ESIMS }\end{array}$ & $\begin{array}{l}\text { Rt } \\
\text { (min.) }\end{array}$ & $\begin{array}{l}\operatorname{HR}-M S(m / z) \text { from }[M-H]^{-}, \\
{[M+H]^{+},[M+K]^{+}(\%)}\end{array}$ & $\begin{array}{l}\text { Molecular } \\
\text { formula }\end{array}$ & $\begin{array}{l}\text { Identified compoundsby } \\
\text { ESIMS }\end{array}$ \\
\hline 9.1 & $191.055777[\mathrm{M}-\mathrm{H}]^{-}$ & $\mathrm{C}_{7} \mathrm{H}_{12} \mathrm{O}_{6}$ & quinic acid & 16.1 & $\begin{array}{l}455.0954180[\mathrm{M}+\mathrm{Na}]^{+} \\
431.0992290[\mathrm{M}-\mathrm{H}]^{-}\end{array}$ & $\mathrm{C}_{21} \mathrm{H}_{20} \mathrm{O}_{10}$ & apigenin-7-O- $\beta$-glucoside \\
\hline 9.7 & $353.087674[\mathrm{M}-\mathrm{H}]^{-}$ & $\mathrm{C}_{16} \mathrm{H}_{18} \mathrm{O}_{9}$ & chlorogenic acid & 17.1 & $\begin{array}{l}487.0992980[\mathrm{M}+ \\
\mathrm{K}]^{+} 447.1293680[\mathrm{M}-\mathrm{H}]^{-}\end{array}$ & $\mathrm{C}_{22} \mathrm{H}_{24} \mathrm{O}_{10}$ & $\begin{array}{l}\text { 6'-methoxy-2',4- } \\
\text { dihydroxychalcone -4'-O- } \beta \text {-D- } \\
\text { glucoside }\end{array}$ \\
\hline 10.2 & $193.050282[\mathrm{M}-\mathrm{H}]^{-}$ & $\mathrm{C}_{10} \mathrm{H}_{10} \mathrm{O}_{4}$ & ferulic acid & 18.6 & $269.0449150[\mathrm{M}-\mathrm{H}]^{-}$ & $\mathrm{C}_{15} \mathrm{H}_{10} \mathrm{O}_{5}$ & Apigenin \\
\hline $\begin{array}{l}13.9 / \\
15.0 / \\
15.6\end{array}$ & $529.135391[\mathrm{M}-\mathrm{H}]^{-}$ & $\mathrm{C}_{25} \mathrm{H}_{24} \mathrm{O}_{12}$ & $\begin{array}{l}\text { Mixture of three dicaffeoyl } \\
\text { quinic acid }\end{array}$ & 19.5 & $287.0919010[\mathrm{M}-\mathrm{H}]^{-}$ & $\mathrm{C}_{16} \mathrm{H}_{16} \mathrm{O}_{5}$ & $\begin{array}{l}\text { 6'-methoxy-2',4, 4'- } \\
\text { trihydroxychalcone }\end{array}$ \\
\hline $\begin{array}{l}15.7 / \\
16.3 / \\
16.6\end{array}$ & $529.135391[\mathrm{M}-\mathrm{H}]^{-}$ & $\mathrm{C}_{26} \mathrm{H}_{26} \mathrm{O}_{1}$ & $\begin{array}{l}\text { Mixture of thtree dicaffeoyl } \\
\text { quinic acid methyl ether }\end{array}$ & 21.4 & $301.2166660[\mathrm{M}-\mathrm{H}]^{-}$ & $\mathrm{C}_{20} \mathrm{H}_{30} \mathrm{O}_{2}$ & $\begin{array}{l}\text { kaur-16-en-18-oic acid } \\
(5 \beta, 8 a, 9 \beta, 10 a, 13 a)\end{array}$ \\
\hline
\end{tabular}

summary of twenty two of the computed molecular descriptors used to assess the drug-likeness profiles of the isolated metabolites have been summarized in Table 2 . These include the \#stars or 'drug-likeness' parameter, the molecular weight (MW), the solvent accessible surface area (SASA), along with its hydrophobic component (FOSA) and hydrophilic component (FISA), the molecular volume, the number of hydrogen bond acceptors (HBA) and donors (HBD), the $n$-octanol/water partition coefficient $(\log \mathrm{P})$, the solubility parameter $(\log S)$, the predicted $\mathrm{IC}_{50}$ values for the blockage of the human-ether-a-go-go potassium ion (HERG $\mathrm{K}^{+}$) channels (logHERG), predicted permeability of Caco-2 cells, the blood-brain barrier partition coefficient (log $\mathrm{BB})$, permeability of Madin-Darby canine kidney (MDCK) monolayers, skin permeability $\left(\log K_{\mathrm{p}}\right)$, the number of predicted primary metabolites (\#metab), the binding affinity to human serum albumin $\left(\log \mathrm{K}_{\mathrm{HSA}}\right)$, the percentage human oral absorption (PHOA), the number of violations of Lipinski's 'Rule of Five' (Ro5) and Jorgensen's 'Rule of Three' (Ro3) and the polar surface area (PSA). The range of values of each parameter for $95 \%$ of known drugs have been given beneath Table 2. Five of these compounds (1, 2, 3, 5 and 12) showed \#star = 0, which indicates that all the computed parameters fell within the recommended range for $95 \%$ of known drugs. Meanwhile, compounds 4 and 6 showed only \#star=1. An overall ADME-compliance score, drug-likeness parameter (indicated by \#stars), was used to assess the pharmacokinetic profiles of the isolated compounds. The \#stars parameter indicates the number of property descriptors computed by QikProp [46], which fall outside the optimum range of values for $95 \%$ of known drugs. The methods implemented were developed by Jorgensen et al. [47-49] (Table 5).

\section{Materials and methods}

\section{General methods}

Silica gel (Merck, 63-200 $\mu \mathrm{m}$ ) and Sephadex LH-20 (Supelco) were used for column chromatography. Fractions were monitored by TLC using precoated silica gel plates $60 \mathrm{~F}_{254}$ (Merck). Spots were visualized by heating silica gel plates sprayed with vanillin- $\mathrm{H}_{2} \mathrm{SO}_{4}$ in $\mathrm{MeOH}$. The ${ }^{1} \mathrm{H}$ and ${ }^{13} \mathrm{C}$ NMR spectra were recorded on a Varian Mercury 300 spectrometer at 300.22 and $75.50 \mathrm{MHz}$, respectively. ${ }^{1} \mathrm{H}$ and $2 \mathrm{D} \mathrm{NMR}$ spectra were recorded on a Varian VNMRS 600 system operating at a proton NMR frequency of $599.83 \mathrm{MHz}$ equipped with a $5 \mathrm{~mm}$ inverse detection cryoprobe using standard CHEMPACK 4.1 pulse sequences (COSY, ROESY, 1DNOESY, HSQCAD, HMBCAD) implemented in Varian VNMRJ 2.2C spectrometer software. Chemical shifts were referenced to internal TMS $\left(\delta=0 \mathrm{ppm},{ }^{1} \mathrm{H}\right)$ and $\mathrm{CDCl}_{3}(\delta=77.0 \mathrm{ppm}$, $\left.{ }^{13} \mathrm{C}\right)$ or $\mathrm{CD}_{3} \mathrm{OD}\left(\delta=49.0 \mathrm{ppm},{ }^{13} \mathrm{C}\right)$, respectively. The high resolution ESI mass spectra were obtained from a Bruker Apex III Fourier transform ion cyclotron resonance (FTICR) mass spectrometer (Bruker Daltonics, Billerica, USA) equipped with an Infinity ${ }^{\mathrm{TM}}$ cell, a 7.0 Tesla superconducting magnet (Bruker, Karlsruhe, Germany), an RF-only hexapole ion guide and an external APOLLO electrospray ion source (Agilent, off axis spray, voltages: endplate, $-3.700 \mathrm{~V}$; capillary, $-4.200 \mathrm{~V}$; capillary exit, $100 \mathrm{~V}$; skimmer 1, $15.0 \mathrm{~V}$; skimmer 2, $10.0 \mathrm{~V}$ ). Nitrogen was used as drying gas at $150{ }^{\circ} \mathrm{C}$. The sample solutions were introduced continuously via a syringe pump with a flow rate of $120 \mu \mathrm{l} / \mathrm{h}$. All data were acquired with $512 \mathrm{k}$ data points and zero filled to $2048 \mathrm{k}$ by averaging 32 scans. The XMASS Software (Bruker, Version 6.1.2) was used for evaluating the data. The positive ion ESI mass spectra and the collision-induced dissociation (CID) mass spectra were obtained from a TSQ Quantum Ultra AM system 
Table 5 Computed molecular descriptors for the assessment of the DMPK profiles of the major isolated metabolites and the recommended range for $95 \%$ of known drugs

\begin{tabular}{|c|c|c|c|c|c|c|c|c|c|c|c|}
\hline Metabolite & ${ }^{a_{\#} \text { stars }}$ & ${ }^{b} \mathrm{CNS}$ & ${ }^{c} \mathrm{MW}$ (Da) & ${ }^{d}$ SASA & ${ }^{e} \mathrm{FOSA}$ & ${ }^{f} \mathrm{FISA}$ & ${ }^{9}$ volume & ${ }^{h} \mathrm{HBD}$ & ${ }^{i} \mathrm{HBA}$ & ${ }^{j} \log P$ & ${ }^{k} \log S$ \\
\hline 1 & 0 & -1 & 286.3 & 506.7 & 157.9 & 145.1 & 880.6 & 2 & 5 & 1.9 & -3.3 \\
\hline 2 & 0 & -2 & 286.3 & 548.9 & 110.0 & 181.5 & 920.6 & 2 & 4 & 2.3 & -3.4 \\
\hline 3 & 0 & -2 & 270.2 & 491.3 & 0 & 200.4 & 827.7 & 2 & 4 & 1.7 & -3.4 \\
\hline 4 & 1 & -2 & 448.4 & 734.5 & 249.3 & 263.0 & 1311.1 & 5 & 13 & 0.4 & -3.1 \\
\hline 5 & 0 & -2 & 448.4 & 683.4 & 261.7 & 245.6 & 1264.2 & 5 & 14 & -0.1 & -3.0 \\
\hline 6 & 1 & -1 & 304.5 & 523.0 & 419.7 & 77.8 & 1007.6 & 1 & 2 & 4.8 & -4.9 \\
\hline 12 & 0 & -2 & 284.3 & 504.1 & 26.4 & 215.2 & 856.2 & 3 & 4 & 1.5 & -3.2 \\
\hline 13 & 5 & -2 & 516.5 & 839.4 & 183.4 & ${ }^{*} 386.8$ & 1495.9 & ${ }^{*} 7$ & 11 & 1.1 & -4.5 \\
\hline Metabolite & 'logHERG & ${ }^{m}$ Caco-2 & ${ }^{n} \log B B$ & ${ }^{\circ} \mathrm{MDCK}$ & ${ }^{P} \log K_{p}$ & ${ }^{9}$ \#metab & ${ }^{r} \log K_{H S A}$ & ${ }^{5} \mathrm{PHOA}$ & ${ }^{t}$ Ro5 & ${ }^{u}$ Ro3 & ${ }^{v}$ PSA \\
\hline 1 & -4.49 & 417.0 & -0.9 & 192.2 & -3.2 & 5 & -0.06 & 84.7 & 0 & 0 & 83.4 \\
\hline 2 & -5.45 & 188.1 & -1.7 & 81.3 & -3.2 & 4 & -0.04 & 80.9 & 0 & 0 & 93.5 \\
\hline 3 & -5.06 & 124.7 & -1.4 & 52.1 & -3.9 & 3 & -0.01 & 74.4 & 0 & 0 & 101.0 \\
\hline 4 & ${ }^{*}-6.01$ & 31.8 & -3.1 & 11.9 & -4.2 & 7 & -0.79 & 43.2 & 1 & 1 & 168.1 \\
\hline 5 & -5.17 & 46.5 & -2.4 & 17.9 & -4.6 & 8 & -0.71 & 56.4 & 0 & 1 & 162.1 \\
\hline 6 & -0.99 & 459.4 & -0.1 & 271.4 & -2.8 & 2 & 0.79 & ${ }^{*} 100$ & 0 & 0 & 42.7 \\
\hline 12 & -4.97 & 90.3 & -1.6 & 36.8 & -4.2 & 5 & -0.10 & 70.5 & 0 & 0 & 109.1 \\
\hline 13 & -4.99 & ${ }^{*} 0.5$ & ${ }^{*}-4.9$ & ${ }^{*} 0.2$ & -6.3 & 6 & -0.63 & 0 & 3 & 1 & 235.6 \\
\hline
\end{tabular}

*Property which falls outside the recommended range for $95 \%$ of known drugs; ${ }^{a}$ Number of computed properties which fall outside the required range for $95 \%$ of known drugs (recommended range 0 to 5 ); ${ }^{b}$ Activity in the central nervous system in the scale -2 (inactive) to +2 (active); ${ }^{c}$ Molar weight (range for $95 \%$ of drugs: $130-725 \mathrm{Da}$ ); ${ }^{d}$ The solvent accessible surface area (recommended range 300.0 to $1000.0 \AA^{2}$ ); ${ }^{e}$ The hydrophobic component of the solvent accessible surface area (recommended range 0.0 to $750.0 \AA^{2}$ ); The hydrophilic component of the solvent accessible surface area (recommended range 7.0 to $330.0 \AA^{2}$ ); ${ }^{g}$ Total volume of molecule enclosed by solvent-accessible molecular surface, in $\AA^{3}$ (probe radius $1.4 \AA$ ) (range for $95 \%$ of drugs: 500 to $2000 \AA^{3}$ ); ${ }^{h}$ Number of hydrogen bonds donated by the molecule (range for $95 \%$ of drugs: 0 to 6); ${ }^{i}$ Number of hydrogen bonds accepted by the molecule (range for $95 \%$ of drugs: $2-20$ ); ${ }^{j}$ Logarithm of partitioning coefficient between $n$-octanol and water phases (range for $95 \%$ of drugs: -2 to 6.5 ); ${ }^{k}$ The predicted aqueous solubility, with $\mathrm{S}$ in mol/dm ${ }^{3}$ (range for $95 \%$ of drugs: -6.5 to 0.5 ); 'Predicted IC 50 value for blockage of HERG $\mathrm{K}^{+}$channels (concern <-5); ${ }^{m}$ Predicted apparent Caco-2 cell membrane permeability in Boehringer-Ingelheim scale, in nm/s (range for $95 \%$ of drugs: < 5 low, $>500$ high); ${ }^{n}$ Logarithm of predicted blood/brain barrier partition coefficient (range for $95 \%$ of drugs: -3.0 to 1.0 ); ${ }^{\circ}$ The predicted apparent MDCK permeability in $\mathrm{nm} / \mathrm{s}\left(<25\right.$ poor, $>500$ great); ${ }^{p}$ The predicted skin permeability (range for $95 \%$ of drugs: -8.0 to -1.0$) ;{ }^{q}$ Number of likely metabolic reactions (range for $95 \%$ of drugs: $1-8$ ); ' Logarithm of predicted binding constant to human serum albumin (range for $95 \%$ of drugs: -1.5 to 1.5 ); ${ }^{5}$ The predicted percentage human oral absorption ( $>80 \%$ high, $<25 \%$ poor); ${ }^{t}$ Number of violations of Lipinski's 'Rule of Five' (Recommended maximum 4); ${ }^{u}$ Number of violations of Jorgensen's 'Rule of Three' (Recommended maximum 3); 'Van der Waals surface area of polar nitrogen and oxygen atoms (range for $95 \%$ of drugs: 7.0 to $200.0 \AA^{2}$ )

equipped with a hot ESI source (HESI, electrospray voltage $3.0 \mathrm{kV}$, sheath gas: nitrogen; vaporizer temperature: $50{ }^{\circ} \mathrm{C}$; capillary temperature: $250{ }^{\circ} \mathrm{C}$; The MS system is coupled with a Surveyor Plus micro-HPLC (Thermo Electron), equipped with a RP18 column $(5 \mu \mathrm{m}, 150 \times 1 \mathrm{~mm}$, Hypersil GOLD, Thermo Scientific). For the HPLC a gradient system was used starting from $\mathrm{H}_{2} \mathrm{O}: \mathrm{CH}_{3} \mathrm{CN}$ 90:10 (each of them containing $0.2 \% \mathrm{HOAc}$ ) to 5:95 within $15 \mathrm{~min}$ and then hold on $5 \%$ for further $30 \mathrm{~min}$; flow rate $70 \mu \mathrm{l} / \mathrm{min}$. Sterols were determined by GC-MS (Voyager/ Trace GC 2000, Thermo Quest CE Instruments): $70 \mathrm{eV}$ EI, source temp. $200{ }^{\circ} \mathrm{C}$; column ZB-5 (Phenomenex, $30 \mathrm{~m} \times 0.25 \mathrm{~mm}, 0.25 \mu \mathrm{m}$ film thickness); inj. temp. $250^{\circ}$ C, interface temp. $300{ }^{\circ} \mathrm{C}$; carrier gas He, flow rate $1.0 \mathrm{ml} /$ min, constant pressure mode; splitless injection, column temp. program: $60{ }^{\circ} \mathrm{C}$ for $1 \mathrm{~min}$, then raised to $300{ }^{\circ} \mathrm{C}$ at a rate of $10{ }^{\circ} \mathrm{C} / \mathrm{min}$ to $290{ }^{\circ} \mathrm{C}$ for $15 \mathrm{~min}$.

\section{Plant material}

The plant materials were collected and identified by Elias Ndive, a botanist from Limbé Botanic Garden, on March
2009 near the town of Buea on the eastern slopes of Mount Cameroon in the South West Province of Cameroon. Voucher specimens (H. foetidum (L.) Moench: SCE2463, H. mechowianum Klatt: SCE2467) are deposited in the Herbarium of Limbé Botanic Garden.

\section{Extraction and isolation}

Leaves and flowers of $H$. foetidum and leaves of $H$. mechowianum were extracted exhaustively with $90 \%$ methanol for a period of $72 \mathrm{~h}$. The solvent was removed by evaporation under reduced pressure. From the crude flower extract of $H$. foetidum, by purification with successive column and preparative TLC chromatography on silica gel using a chloroform/methanol gradient systems, the compounds 7,4' -dihydroxy-5-methoxy-flavanone (1) and kaur16-en-18-oic acid (6) were obtained while the compounds 6 '-methoxy-2',4,4' -trihydroxychalcone (helichrysetin) (2), 6 '-methoxy-2',4-dihydroxychalcone-4' - $O$ - $\beta$-D-glucoside (3), apigenin (4), apigenin-7- $O-\beta$-D-glucoside (5) were isolated from the leaves and flowers of $H$. Foetidum. 
The aqueous residue of the crude extract of $H$. mechowianum leaves was partitioned successively with $n$-heptane and ethyl acetate. The $n$-heptane and the ethyl acetate extracts were further purified by silica gel column chromatography using $n$-hexane/ethyl acetate gradient systems resulting in the isolation of a phytosterol fraction and of 3,5,7-trihydroxy-8-methoxyflavone (12), respectively. The water fraction was further separated using Diaion HP20 eluted with water, methanol, ethyl acetate and acetone followed by chromatography of the methanol fraction on Sephadex LH20 to give 4,5-dicaffeoyl quinic acid (13). The crude extracts of $H$. foetidum and $H$. mechowianum were analyzed by LC-ESI-MS, MS/MS and FTICR-HRMS.

7,4'-dihydroxy-5-methoxy-flavanone (1): ${ }^{1} \mathrm{H} \quad \mathrm{NMR}$ $\left(\right.$ DMSO- $\left.\mathrm{d}_{6}\right): \delta 9.60(1 \mathrm{H}$, brs, $\mathrm{OH}), 7.28\left(2 \mathrm{H}, \mathrm{d}, 8.6, \mathrm{H} 2{ }^{\prime} /\right.$ 6'), 6.77 (2H, d, 8.6, H3'/5'), $6.04(1 \mathrm{H}, \mathrm{d}, 2.2, \mathrm{H} 6), 5.94$ $(1 \mathrm{H}, \mathrm{d}, 2.2, \mathrm{H} 8), 5.32(1 \mathrm{H}, \mathrm{dd}, 12.6 / 2.9, \mathrm{H} 2), 3.72(3 \mathrm{H}, \mathrm{s}$, OMe), 2.98 (1H, dd, 16.3/12.6, H3b), ca. 2.5 (1H, m, superimposed by DMSO, H3a). ${ }^{13} \mathrm{C}$ NMR (DMSO-d $\left.{ }_{6}\right) \delta$ 79.0(C2), 42.4(C3), 196.3(C4), 163.0(C5),104.6(C6),167.1 (C7),95.4(C8),162.8(C9),101.6(C10), 129.6(C1'), 127.6(C2' /C6'), 115.0(C3'/C5'), 157.6(C4'), 55.4 (OMe).

6'-methoxy-2',4,4'-trihydroxychalcone (2): ${ }^{1} \mathrm{H}$ NMR $\left(\mathrm{MeOD}+\mathrm{CDCl}_{3}\right): \delta 7.73(1 \mathrm{H}, \mathrm{d}, 16.0, \mathrm{H \alpha}), 7.68(1 \mathrm{H}, \mathrm{d}$, 16.0, H 3$), 7.49$ (2H, d, 8.6, H2/6), 6.84 (2H, d, 8.6, H3/ 5), $5.99\left(1 \mathrm{H}, \mathrm{d}, 2.2, \mathrm{H} 3^{\prime}\right), 5.96\left(1 \mathrm{H}, \mathrm{d}, 2.2, \mathrm{H} 5^{\prime}\right), 3.93$ (OMe) ${ }^{13} \mathrm{C} \mathrm{NMR}\left(\mathrm{MeOD}+\mathrm{CDCl}_{3}\right): \delta 193.6(\mathrm{C}=\mathrm{O})$,

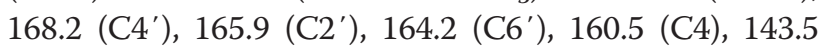
(CH- $\beta), 131.0(\mathrm{C} 2 / 6), 128.0(\mathrm{C} 1), 125.2(\mathrm{CH}-\alpha), 116.6$ (C3/5), 106.3 (C1'), 96.9 (C5'), 92.2 (C3'), 56.2 (OMe).

6'-methoxy-2',4-dihydroxychalcone-4'-O- $\beta$-D-glucoside (3): ${ }^{1} \mathrm{H}$ NMR (MeOD): $\delta 7.72(1 \mathrm{H}, \mathrm{s}, \mathrm{H} \alpha), 7.71(1 \mathrm{H}, \mathrm{s}$, $\mathrm{H} \beta), 7.51$ (2H, d, 8.7, H2/6), 6.83 (2H, d, 8.7, H3/5), 6.31 $\left(1 \mathrm{H}, \mathrm{d}, 2.2, \mathrm{H} 3^{\prime}\right), 6.24\left(1 \mathrm{H}, \mathrm{d}, 2.2, \mathrm{H} 5^{\prime}\right), 3.95(3 \mathrm{H}, \mathrm{s}$, $\mathrm{OMe})$, glucose moiety: $\delta 4.99\left(1 \mathrm{H}, \mathrm{d}, 7.5, \mathrm{H} 1^{\prime \prime}\right)$, 4.24(H2"), 3.61(H3"), 3.60(H4"), 3.53(H5"), 3.80-3.90 (H6"), ${ }^{13} \mathrm{C}$ NMR (MeOD) $\delta 193.6(\mathrm{C}=\mathrm{O}), 168.2$ (C4'), 165.9 (C2'), 164.2 (C6'), 160.5 (C4), 143.5 (CH- $\beta$ ), 131.0 (C2/6), 128.0 (C1), 125.2 (CH- $\alpha), 116.6$ (C 3/5), 106.3 (C1'), 96.9 (C5'), 92.2 (C3'), 56.2 (OMe) glucose moiety: $\delta$ 73.7(C1"), 71.0(C2"), 77.2(C3"), 78.9(C4"), 79.8(C5"), 60.6(C6"),

apigenin (4): ${ }^{1} \mathrm{H}$ NMR (DMSO- $\left.\mathrm{d}_{6}\right): \delta 12.96(1 \mathrm{H}, \mathrm{s}$, $\mathrm{OH}), 7.93\left(2 \mathrm{H}, \mathrm{d}, 8.8, \mathrm{H} 2^{\prime} / 6^{\prime}\right), 6.93\left(2 \mathrm{H}, \mathrm{d}, 8.8, \mathrm{H} 3^{\prime} / 5^{\prime}\right)$, $6.78(1 \mathrm{H}, \mathrm{s}, \mathrm{H} 3), 6.48(1 \mathrm{H}, \mathrm{d}, 2.0, \mathrm{H} 8), 6.19(1 \mathrm{H}, \mathrm{d}, 2.0$, H6). ${ }^{13} \mathrm{C}$ NMR (DMSO-d 6 ): $\delta 180.4$ (C4), 164.94 (C5), 164.4 (C2), 162.6 (C4'), 160.7 (C9), 160.1 (C7), 129.3 (C2'/ C6'), 123.1 (C1'), 117.0 (C3'/ C5'), 109.3 (C10), 106.5 (C3), 104.8 (C6), 99.3 (C8).

apigenin-7-O- $\beta$-D-glucoside (5): ${ }^{1} \mathrm{HNMR}\left(\mathrm{CD}_{3} \mathrm{OD}, 500\right.$ $\mathrm{MHz}$ ): aglycon moiety: $\delta 7.83\left(2 \mathrm{H}, \mathrm{d}, 8.8, \mathrm{H}-2^{\prime} / \mathrm{H}-6^{\prime}\right)$, $6.92\left(2 \mathrm{H}, \mathrm{d}, 8.8, \mathrm{H}-3^{\prime} / \mathrm{H}-5^{\prime}\right), 6.83$ (1H,d, $\left.2.1 \mathrm{~Hz}, \mathrm{H}-6\right)$, $6.71(1 \mathrm{H}, \mathrm{d}, 2.1 \mathrm{~Hz}, \mathrm{H}-8), 6.58(1 \mathrm{H}, \mathrm{s}, \mathrm{H}-3)$, glucose moiety: $\delta 4.90$ (1H,d, 7.6, H-1"'), 3.50-3.25 (4H,m,H-2", 3 ", 4", 5"), 3.87 (1H,dd, 11.9/ 2.2, H-6 $\left.{ }^{\prime \prime}\right), 3.73$ (1H,dd,11.9/ 5.4, H-6a" ${ }^{13} \mathrm{C}$ NMR (DMSO-d 6 ): $\delta 182.0$ (C4), 164.3 (C5), 163.0 (C2), 161.5 (C4'), 161.1 (C9), 157.0 (C7), 128.7 (C2'/C-6'), 121.0 (C1'), 116.0 (C3'/ C5'), 105.4 (C10), 103.1 (C3), 99.9 (C6), 99.6 (C8), glucose moiety: $\delta 105.1$ (C1"), 78.6 (C5"), 77.5 (C3"), 74.8 (C2"), 71.8(C4"), 62.6 (C6")

kaur-16-en-18-oic acid (6): ${ }^{1} \mathrm{H}$ NMR $\left(\mathrm{CDCl}_{3}\right): \delta 4.79$ (1H, s, H17), 4.73 (1H, s, H17), 2.63 (1H,brs), $2.15(1 \mathrm{H}$, brd,13.9), 2.05 (2H, d, 2.0), $1.24(3 \mathrm{H}, \mathrm{s}, \mathrm{Me}), 0.95(3 \mathrm{H}, \mathrm{s}$, $\mathrm{Me}){ }^{13} \mathrm{C} \mathrm{NMR}\left(\mathrm{CDCl}_{3}\right): \delta 184.1(\mathrm{C}=\mathrm{O}), 155.9(\mathrm{C}), 103.0$ $\left(\mathrm{CH}_{2}\right), 57.0(\mathrm{CH}), 55.1(\mathrm{CH}) 48.9\left(\mathrm{CH}_{2}\right), 44.2(\mathrm{C}), 43.8$ $(\mathrm{CH}), 43.7(\mathrm{C}), 41.3\left(\mathrm{CH}_{2}\right), 40.7\left(\mathrm{CH}_{2}\right), 39.7\left(\mathrm{CH}_{2}\right), 39.6$ (C), $37.8\left(\mathrm{CH}_{2}\right), 33.1\left(\mathrm{CH}_{2}\right), 28.9\left(\mathrm{CH}_{3}\right), 21.8\left(\mathrm{CH}_{2}\right), 19.1$ $\left(\mathrm{CH}_{2}\right), 18.4\left(\mathrm{CH}_{2}\right), 15.6\left(\mathrm{CH}_{3}\right)$.

3,5,7-trihydroxy-8-methoxyflavone (12): ${ }^{1} \mathrm{H}$ NMR (CD $\left.\mathrm{Cl}_{3}\right): \delta 11.49(1 \mathrm{H}, \mathrm{s}, \mathrm{OH}), 8.24(2 \mathrm{H}, \mathrm{d}, 7.1), 7.60-7.49$ $(3 \mathrm{H}, \mathrm{m}), 6.70(1 \mathrm{H}, \mathrm{s}, \mathrm{OH}), 6.46(1 \mathrm{H}, \mathrm{s}, \mathrm{OH}), 6.33(1 \mathrm{H}, \mathrm{s}$, H6), 4.05 (3H, s, OMe). ${ }^{13} \mathrm{C}$ NMR $\left(\mathrm{CDCl}_{3}\right): \delta 175.6$ (C = O), 156.6 (C), 155.4 (C), 148.0 (C), 144.9 (C), 136.5 (C), $130.7(\mathrm{C}), 130.4(\mathrm{CH}), 128.8(2 \mathrm{CH}), 127.5(2 \mathrm{CH})$, $126.9(\mathrm{C}), 98.2(\mathrm{CH}), 61.9$ (OMe).

4,5-dicaffeoyl quinic acid (13): ${ }^{1} \mathrm{H}$ NMR (MeOD): $\delta$ 7.585/7.507 (1H, d, 16.0, H7'/7"), 7.014/6.994 (1H, d, 2.0, H2'/2"), 6.905/6.890 (1H, dd, 8.2/2.0, H6'/6"), 6.739/6.733 (1H, d, 8.2, 5'/5"), 6.273/6.190 (1H, d, 16.0, H8'/8"), 5.661 (1H, m, H5) 5.108 (1H, dd, 9.8/3, H4), 4.337 (1H, d, 3, H3), 2.284 (1H, brdd, $14.12 .1, \mathrm{H} 2 \mathrm{a})$, ca. 2.22 (2H, H6), 2.046 (1H, brd, 12.5, H2a) ${ }^{13} \mathrm{C}$ NMR (MeOD): $\delta 178.9$ (C7), 168.6/168.4 (C9'/9"), 149.6 (C4'/ $\left.4^{\prime \prime}\right), 147.6 / 147.4$ (C7'/7"), 146.75/146.73 (C3'/3"), 127.7/ 127.6 (C1'/1"), 123.1 (C6'/6"), 116.4 (C5'/5"), 115.1 (C2'/ 2"), 114.8 (C8'/8"), 76.9 (C1), 76.6 (C4), 70.2 (C3), 69.3 (C5), 40.2 (C6), 38.7 (C2).

\section{Bioassays \\ Protease inhibition assay \\ Procedure}

Initially the extracts or purified constituents were dissolved in DMSO and the dilutions of samples tested were made in the respective buffer for each enzyme, i.e., $0.1 \mathrm{M}$ sodium phosphate ( $\mathrm{pH} 7.5)$ for subtilisin and $0.1 \mathrm{M}$ sodium acetate $(\mathrm{pH} 4.4)$ for pepsin. Samples $(0.01-50 \mu \mathrm{g} / \mathrm{ml})$ were pre-incubated with subtilisin (37 $\mathrm{nM})$ or pepsin $(1.7 \mathrm{nM})$ for $30 \mathrm{~min}$ and then, transferred to a black opaque microplate. The substrate EDANS-DABCYL $(2 \mu \mathrm{M})$, prepared in the specific buffer for each protease, were automatically injected. The final volume was $100 \mu \mathrm{l}$. Experiments were performed separately for each protease, which was prepared at the day of experiment. Reads were made for a period of $5 \mathrm{~min}$, with $1 \mathrm{~min}$ intervals, and temperature controlled at $37{ }^{\circ} \mathrm{C}$. The mean, standard deviation and relative 
standard deviation (RSD) of triplicates and the percentage of inhibition were calculated using the final fluorescence intensity measured.

\section{Reagents}

Pepsin from porcine gastric mucosa, Recombinant Type VIII Subtilisin Carlsberg, Arg-Glu-(EDANS)-Ser-Gln-AsnTyr-Pro-Ile-Val-Gln-Lys-(DALBCYL)-Arg fluorogenic substrate (EDANS-DABCYL), DMSO spectrophotometric grade were from Sigma-Aldrich, Sao Paulo, Brazil. Fluorescence bioassay data were collected with a multi detection microplate reader Synergy ${ }^{\mathrm{TM}}$ HT (Bio-Tek ${ }^{\circ}$ Instruments Inc., Winooski, Vermont, USA), with $360 \mathrm{~nm}$ excitation and $460 \mathrm{~nm}$ emission filters, and analyzed using $\mathrm{KC} 4$ software (Bio-Tek ${ }^{\circ}$ Instruments) and a Microsoft Windows XP.

\section{Antibacterial assay}

The antibacterial activity against a fluorescent Bacillus subtilis [50] was determined with a fluorescence based antibacterial growth inhibition assay. The fluorescence was measured on a microtiter plate reader GENios Pro (Fa. Tecan, excitation $510 \mathrm{~nm}$; emission $535 \mathrm{~nm}$ ). The Bacillus subtilis strain 168 ( $\left.\mathrm{P}_{\mathrm{AbrB}}-\mathrm{IYFP}\right)$ was maintained on TY (tryptone-yeast extract) medium supplemented with $1 \%$ Bacto-tryptone, $0.5 \%$ Bacto-yeast extract, $1 \%$ $\mathrm{NaCl}$ and Chloramphenicol $(5 \mu \mathrm{g} / \mathrm{ml})$. Erythromycin was used as positive control for growth inhibition.

\section{Antifungal assay}

The antifungal activity against the phytopathogenic fungus Cladosporium cucumerinum was tested by bioautography on silica gel plates [51] in concentrations of 50, 100,200 and $400 \mu \mathrm{g} / \mathrm{cm}$. Amphotericine B was used as positive control for growth inhibition.

\section{Cytotoxicity assay}

The cytotoxicity was determined by XTT method, using the Cell Proliferation Kit II (Roche). The human prostate cancer cell line PC-3 was maintained in RPMI 1640 medium supplemented with $10 \%$ fetal bovine serum, $1 \%$ L-alanyl-L-glutamin $(200 \mathrm{mM}), 1 \%$ penicillin/streptomycin and 1,6 \% hepes $(1 \mathrm{M})$. For the measurement of cytotoxicity the same medium was used without antibiotics. For PC-3 500 cells/well were seeded overnight into 96-well plates and exposed to serial dilution of each compound for three days.

\section{Molecular modeling}

All molecular modelling was carried out on a Linux workstation running on a $3.5 \mathrm{GHz}$ Intel Core2 Duo processor (Santa Clara, USA). Low energy 3D structures of the thirteen isolated compounds were generated using the MOE software package [52] and the Merck molecular forecefiled [53] and saved in mol2 format. These were initially treated with LigPrep [54], distributed by Schrodinger, Inc (Camberley, UK). This implementation was carried out with the graphical user interface (GUI) of the Maestro software package (New York, USA) [55], using the OPLS force field [56-58]. Protonation states at biologically relevant $\mathrm{pH}$ were correctly assigned (group I metals in simple salts were disconnected, strong acids were deprotonated and strong bases protonated, while topological duplicates and explicit hydrogens were added). A set of the ADMET-related properties (a total of 46 molecular descriptors) were calculated using the QikProp program (New York, USA) [46] running in normal mode. QikProp generates physically relevant descriptors and uses them to perform ADMET predictions. An overall ADME-compliance score, druglikeness parameter (indicated by \#stars), was used to assess the pharmacokinetic profiles of the compounds. The \#stars parameter indicates the number of property descriptors computed by QikProp, which fall outside the optimum range of values for $95 \%$ of known drugs. The methods implemented were developed by Jorgensen et al. [47-49].

\section{Conclusion}

Eight known compounds have been identified from the extracts of two species from the genus Helichrysum (Compositae) harvested from the South West of Cameroon (Central Africa). The results showed that the flavonoid glycosides $(3,5)$ from $H$. foetidum exhibited protease inhibition, while the compound (13) from $H$. mechowianum contribute to the stomach protecting effects. In addition, the antibacterial and antifungal activities of compound (6) was demonstrated by the fact that it was found to possess a potent inhibitor effect against the tested microorganisms. The differential bioactivities and determined constituents support the traditional use of the species. Molecular modelling studies showed that five of the isolated compounds showed physicochemical properties that completely within the recommended range for more that $95 \%$ of known drugs, while two compounds have only one violation.

\section{Additional file}

Additional file 1: Figure S1. LC-MS of Helichrysum mechowianum, $\mathrm{MeOH}$ fraction positive and negative ion. Figure S2. LC-MS of Helichrysum foetidum leaf extract positive and negative ion.

Competing interests

The authors declare that they have no competing interests.

\section{Authors' contributions}

LW, VSB, JCN, FK, OJF and EMM designed the experiments, FAEM, ABN, LN carried out the experiments, while FNK did the molecular modeling. All authors read and approved the final manuscript. 


\section{Author details}

${ }^{1}$ Department of Pharmacy, University of Douala, Douala, P.O. Box 812, Cameroon. ${ }^{2}$ Department of Chemistry, University of Douala, Douala, P.O. Box 567, 24157, Cameroon. ${ }^{3}$ Química, Departamento de Química Orgânica, Núcleo de Bioensaio, Biossíntese e Ecofisiologia de Produtos Naturais-NuBBE, Universidade Estadual Paulista (UNESP) Instituto de Rua Prof. Francisco Degni s/n, Araraquara, São Paulo 14.800-900, Brazil. ${ }^{4}$ Department of Bioorganic Chemistry, Leibniz Institute of Plant Biochemistry, Weinberg 3, D-06120 Halle (Saale), Germany. ${ }^{5}$ Chemical and Bioactivity Information Centre, Department of Chemistry, University of Buea, P. O. Box 63, Buea, Cameroon

Received: 14 May 2015 Accepted: 19 May 2015

Published online: 30 May 2015

\section{References}

1. Pand Lesotho. Flora Publications Trust, Durban, South Africa.ooley, E., 2003. Mountain flowers. A Field Guide to the Flora of the Drakensberg

2. Tchoumbougnang F, Sameza ML, Jazet Dongmo PM, Nkouaya MEG, Fekam $\mathrm{BF}$, Ngoko Z, et al. Composition, radical scavenging and antifungal activities of essential oils from 3 Helichrysum species growing in Cameroon against Penicillium oxalicum a yam rot fungi. Afr J Agric Res. 2010;4:121-7.

3. Mathekga ADM, Meyer MJJ, Horn MM, Drewes SE. An acylated phloroglucinol with antimicrobial properties from Helichrysum caespititium. Phytochemistry. 2000;53:93-6.

4. Puyvelde LV, De Kimpe N, Costa J, Munyjabo V, Nyirankuliza S, Hakizamungu E, et al. Isolation of flavonoids and a chalcone from Helichrysum odoratissimum and synthesis of helichrysetin. J Nat Prod. 1989;52:629-33.

5. Boiteau P, Allorge-Boiteau L. Plantes médicinales de Madagascar. Cinquante - huit plantes médicinales. Karthala: ACCT; 1993. p. 46.

6. Litvinenko VI, Popova P, Popova NV, Bubenchikova VN. Medicinal plants and preparations derived from them. Farmaseotychnyi Zhurnal. 1992;53:83-4.

7. Rigano D, Formisano C, Senatore F, Piacente S, Pagano E, Capasso R, et al. Intestinal antispasmodic effects of Helichrysum italicum (Roth) Don ssp. italicum and chemical identification of the active ingredients. J Ethnopharmacol. 2003;150:901-6.

8. Ibara JR, Elion Itou RDG, Ouamba JM, Diatewa M, Gbeassor M, et al. Preliminary evaluation of antiulcerogenic activity of Ceiba pentandra Gaertn. and Helicrysum mechowianum Klatt in rats. J Med Sci. 2007;7:485-8.

9. Sezik E, Yesilada E, Honda G, Takaishi Y, Takeda Y, Tanaka T. Traditional medicine in Turkey X Folk medicine in Central Anatolia. J Ethnopharmacol. 2001;75:95-115.

10. Kambu K. Eléments de phytothérapie comparée; plantes médicinales africaines. Centre de recherches Pédagogiques de. Franç: Kinshasa; 1990. p. 196 p.

11. Bougatsos C, Ngassapa O, Deborah K, Runyoro B, Chinou IB. Chemical composition and in vitro antimicrobial activity of the essential oils of two Helichrysum species from Tanzania. Z Naturforsch C. 2004;59:368-72.

12. Facino RM, Carini M, Franzoi L, Pirola O, Bosisio E. Phytochemical characterization and radical scavenger activity of flavonoids from Helichrysum italicum. Pharmacol Res. 1990;22:709-21.

13. Cubukcu B, Yuksel V. Constituents of Anatolian medicinal plants; flavonoids of Helichrysum armenium. J Nat Prod. 1982;45:137-9.

14. Tomas-Barberan FA, Maillard M, Hostettmann K. Antifungal flavonoids from the leaf surfaces of Helichrysum nitens and from the stem bark of Erythrina berteroana. Prog Clin Biol Res. 1988;280:61-5.

15. Tomas-Barberan FA, Msonthi JD, Hostettmann K. Anti-fungal epicuticular methylated flavonoids from Helichrysum nitens. Phytochemistry. 1988;27:753-5.

16. Iniesta-Sanmartin E, Tomas-Barberan FA, Guirado A, Tomas-Lorente F. Antibacterial flavonoids from Helichrysum picardii and $H$. italicum. Planta Med. 1990;56:648-9.

17. Kakam AMZ, Hussain H, Dongo E, Kouam SF, Schulz B, et al. Cameroonemide A: a new ceramide from Helichrysum cameroonense. J Asian Nat Prod Res. 2010;12:629-33.

18. Kakam AMZ, Franke K, Ndom JC, Dongo E, Ngando Mpondo T. Secondary metabolites from Helichrysum foetidum and their chemotaxonomic significance. Biochem Syst Ecol. 2011:39:166-7.

19. Bohlmann F, Abraham WR. Neue Prenylflavanone aus Helichrysum hypocephalum. Phytochemistry. 1979;18:1851-3.

20. Bohlmann F, Misra LN. New prenylflavanones and chalcones from Helichrysum rugulosum. Planta Med. 1984;50:271-2.
21. Jakupovic J, Zdero C, Grenz M, Tsichritzis F, Lehmann L, Hashemi-Nejad SM, et al. Twenty-one acylphloroglucinol derivatives and further constituents from South African Helichrysum species. Phytochemistry. 1989;28:1119-31.

22. Jakupovic J, Grenz M, Bohlmann F, Mungai GM. 12ß-Hydroxyabieta-7, 13-diene and other constituents from East African Helichrysum species. Phytochemistry. 1990;29:1589-90.

23. Randriaminahy M, Proksch P, Witte L, Wray V. Lipophilic phenolic constituents from Helichrysum species endemic to Madagascar. Z Naturforsch C. 1992:47:10-6.

24. Caffaratti M, Ortega MG, Scarafia ME, Espinar LA, Juliani HR. Prenylated flavanones from Dalea elegans. Phytochemistry. 1994;36:1083-4.

25. Matsumoto J, Fujimoto T, Takino C, Saitoch M, Hano Y, Fukai T, et al. Compounds of Broussonetia papyrifera (L.) Vent. I. Structures of two new isoprenylated flavonols and two chalcone derivatives. Chem Pharm Bull. 1985;33:3250-6

26. Lourens AC, Viljoen AM, van Heerden RR. South African Helichrysum species: A review of the traditional uses, biological activity and phytochemistry. J Ethnopharmacol. 2008;119:630-52.

27. Adjanohoun E, Ahyi MRA, Ake Assi L, Baniakina J, Chibon P, Cusset G, et al. Contribution aux études ethnobotaniques et floristiques en République Populaire du Congo. Paris: ACCT; 1988. p. 605.

28. Lim TK. Ceiba pentandra. Edible Medicinal and Non-Medicinal Plants, vol 1, Fruits, Springer Sci 2012, pp 540-49

29. Ibara JR, Elion Itou RDG, Etou-Essebi A, Ouamba J, Diatewa M, Abena A. Enquête ethnobotanique à propos de plantes médicinales congolaises présumées anti-ulcéreuses. Phytothérapie. 2007;5:118-20.

30. Flausino Jr O, Abissi BM, Vieira J, Santos AG, Silva DH, Cavalheiro A, et al. Protease inhibition activity of extracts from Salicaceae species from Brazilian Cerrado and Atlantic Rain Forest and of an enriched fraction of clerodanediterpenes (casearins). Braz J Pharmacogn. 2009;17:755-8.

31. Ryzhova GL, Kravtsova SS, Matasova SA, Gribel NV, Pashinskii VG, Dychko KA, et al. Chemical and pharmacological properties of the dry extract from Black Birch fungus. Pharmaceut Chem J. 1997;31:551-4.

32. Fabry W, Okomo PO, Ansorg R. Antibacterial activity of East African medicinal plants. J Ethnopharmacol. 1998;60:79-84.

33. Gibbons S. Anti-staphylococcal plant natural products. Nat Prod Rep. 2004;21:263-77.

34. Rios JL, Reico MC. Medicinal plants and antimicrobial activity. J Ethnopharmacol. 2005;100:80-4.

35. Adnan JR, Omar AA, Mahmoud MO, Jaber SM. Flavonoids and terpenoids from Helichrysum forskahlii. Phytochemistry. 2008;69:1910-4.

36. Mottakin AKM, Chowdhury R, Haider MS, Rahman KM, Hasan CM, Rashid MA, et al. Cytotoxicity and antibacterial activity of extractives from Wedelia calendulacea. Fitoterapia. 2004;75:355-9.

37. Ndom JC, Mbafor TJ, Meva'a ML, Kakam AMZ, Arwa SP, Dongo E, et al. New alkamide and ent-kaurane diterpenoid derivatives from Senecio erechtitoides (Asteraceae). Phytochem Lett. 2010;3:201-6.

38. Zapesochnaya GG, Dzyadevich TV, Karasartov BS. Phenolic compounds of Helichrysum italicum. Chem Nat Compd. 1990;26:342-3.

39. Zapesochnaya GG, Kurkin VA, Kudyavtseva TV, Karasartov KS, Cholponbaev KS, Tyukavkina NA, et al. Dicaffeoylquinic acids from Helichrysum italicum and Achillea cartilaginea. Chem Nat Compd. 1992;28:40-4.

40. Lavault M, Richome P. Constituents of Helichrysum stoechas variety olonnense. Chem Nat Compd. 2004;40:118-21.

41. Zhu X, Zhang H, Lo R. Phenolic constituents from the leaf extract of artichoke (Cynaria scolymus L.) and their antimicrobial activities. J Agric Food Chem. 2004:52:7272-8.

42. Karasartov BS, Kurkin VA, Zapesochnaya GG. Coumarins and flavonoids of the flowers of Helichrysum italicum. Khimiya Prirodnykh Soedinenii. 1992:5:577-9.

43. Lin LC, Kuo YC, Chou CJ. Immunomodulatory principles of Dichrocephala bicolor. J Nat Prod. 1999:62:405-8.

44. Mishima S, Inoh Y, Narita Y, Ohta S, Sakamoto T, Araki Y, et al. Identification of caffeoylquinic acid derivatives from Brazilian propolis as constituents involved in induction of granulocytic differentiation of HL-60 cells. Bioorg Med Chem. 2005;13:5814-8.

45. Radette-Hebert ME, Legault J, Lavoie S, Pichette A. Chem Pharm Bull. 2008:56:82-4.

46. Schrödinger Press. QikProp 3.4 User Manual. New York: LLC; 2011.

47. Jorgensen WL, Duffy EM. Prediction of drug solubility from structure. Adv Drug Deliv Rev. 2002;54:355-66. 
48. Duffy EM, Jorgensen WL. Prediction of properties from simulations: free energies of solvation in hexadecane, octanol, and water. J Am Chem Soc. 2000;122:2878-88.

49. Jorgensen WL, Duffy EM. Prediction of drug solubility from Monte Carlo simulations. Bioorg Med Chem Lett. 2000;10:1155-8.

50. Veening JW, Smits WK, Hamoen LW, Jongbloed JDH, Kuipers OP. Visualization of differential gene expression by improved cyan fluorescent protein and yellow fluorescent protein production in Bacillus subtilis. Appl Env Microbiol. 2004;70:6809-15.

51. Gottstein D, Gross D, Lehmann H. Mikrobiotest mit Cladosporium cucumerinum Ell. et. Arth. zum Nachweis fungitoxischer Verbindungen auf Dünnschichtplatten. Archiv für Phytopathologie und Pflanzenschutz. 1982:20:111-6.

52. Chemical Computing Group Inc. Molecular Operating Environment Software. Montreal: Chemical Computing Group Inc; 2010

53. Halgren TA. Merck molecular forcefield. J Comput Chem. 1996;17:490-641.

54. Schrödinger. LigPrep software, version 2.5. New York: LLC; 2011.

55. Schrödinger. Maestro, version 9.2. New York: LLC; 2011.

56. Shivakumar D, Williams J, Wu Y, Damm W, Shelley J, Sherman W, et al. Prediction of absolute solvation free energies using molecular dynamics free energy perturbation and the OPLS force field. J Chem Theory Comput. 2010;6:1509-19.

57. Jorgensen WL, Maxwell DS, Tirado-Rives J. Development and testing of the OPLS all-atom force field on conformational energetics and properties of organic liquids. J Am Chem Soc. 1996;118(45):11225-36.

58. Jorgensen WL, Tirado-Rives J. The OPLS [optimized potentials for liquid simulations] potential functions for proteins, energy minimizations for crystals of cyclic peptides and crambin. J Am Chem Soc

1988;110(6):1657-66

\section{Publish with ChemistryCentral and every scientist can read your work free of charge \\ "Open access provides opportunities to our colleagues in other parts of the globe, by allowing anyone to view the content free of charge." \\ W. Jeffery Hurst, The Hershey Company. \\ - available free of charge to the entire scientific community \\ - peer reviewed and published immediately upon acceptance \\ - cited in PubMed and archived on PubMed Central \\ - yours - you keep the copyright \\ Submit your manuscript here: \\ http://www.chemistrycentral.com/manuscript/<smiles>c1ccccc1</smiles> \\ Chemistry Central}

